\title{
Espalhamento inelástico da luz por portadores no plasma em semicondutores. I Cálculo da Função Dielétrica
}

Light Scattering by Carriers Plasmas in Semiconductors. I Dielectric Function Analysis

\author{
A.V. Andrade-Neto* \\ Departamento de Física - UEFS \\ Campus Universitário, $\mathrm{Km}$ 03, BR 116 \\ Feira de Santana - BA - 44031-460
}

\begin{abstract}
Neste trabalho, utilizando a aproximação de banda parabólica, obtemos expressões analíticas para as partes real e imaginária da função dielétrica longitudinal de um plasma em estado sólido no limite de altas temperaturas, onde podemos substituir a função de Fermi-Dirac por uma distribuição do tipo Maxwell-Boltzmann. Em seguida, analisamos seus casos particulares, casos estático e de altas freqüências, e recuperamos expressões conhecidas na literatura.
\end{abstract}

Palavras-chaves: Função dielétrica, Blindagem, Oscilação de plasma.

In this work we obtain analytical expression for real e imaginary parts for dielectric function for the solid state plasma in the limit case at high temperature, where we can substitute the Fermi-Dirac function by the Maxwell-Boltzmann one. By using the parabolic band approximation and we obtain a general expression that, in our approach, is valid for all values for wavevector $Q$. We analysis the longitudinal dielectric function in two important cases: static and high frequencies.

Key-words: Dielectric function, Screening, Plasma oscillation.

\section{INTRODUÇÃ̃o}

Um plasma é um conjunto neutro de partículas carregadas que interagem entre si via forças coulombianas e que exibe um comportamento coletivo ${ }^{1}$. Por analogia foi cunhada a expressão Plasma em Estado Sólido [1] para designar qualquer conjunto de portadores de cargas intinerantes em um sólido, cuja carga elétrica é neutralizada por uma distribuição uniforme de carga positiva. Tais plasmas são encontrados em metais, semimetais e semicondutores. Este último caso, que denominaremos Plasma em Semicondutores, tem-se tornado uma importante área da física da matéria condensada [1], já que estes sistemas permitem o acesso a um amplo espectro de parâmetros experimentais tais como: freqüência de plasma, freqüência ciclotrônica, energia de Fermi, energia do gap, entre outros, como também permitem o controle da concentração de portadores através da dopagem por impurezas ou por fotoinjeção por laser. No primeiro caso, para um semicondutor tipo $n(p)$, os portadores são apenas elétrons (buracos). Logo, dizemos que trata-se de um plasma simples ou plasma de componente única. No segundo caso temos a presença de ambos os tipos de portadores: elétrons e buracos; assim, falamos em plasma multicomponente ou, mais precisamente, plasma duplo fotoinjetado. Este se forma quando há transições eletrônicas provocadas por radiação laser, por exemplo, da banda de valência para a banda de condução, e resulta numa distribuição de elétrons na banda de condução e buracos na banda de valência, altamente excitados. As

\footnotetext{
*Endereço Eletrônico: aneto@uefs.br

${ }^{1}$ Para definir o plasma mais precisamente é necessário introduzir alguns conceitos que descrevam o comportamento característico do mesmo como, por exemplo, comprimento de blindagem, freqüência de plasma, etc; já que um conjunto de partículas carregadas, eletricamente neutro, nem sempre constitui um plasma.
}

partículas fotoinjetadas (portadores) relaxam sua energia em excesso através do processo de recombinação radiativa, que consiste na recombinação entre um elétron e um buraco com emissão de radiação eletromagnética; e a dissipação se dá, principalmente, por intermédio da interação com os modos normais de vibração da rede (interação portadorfônon). O plasma duplo fotoinjetado em semicondutores é, assim, um sistema fortemente afastado do equilíbrio, que tem merecido grande atenção devido, por um lado, ao grande interesse científico intrínseco e, por outro, pelo interesse tecnológico/industrial/comercial. Plasma em semicondutores fotoinjetado altamente excitado é tratado em [2]. Neste trabalho estamos interessados em um plasma simples (semicondutor dopado tipo $n$, por exemplo).

O espalhamento inelástico da luz tem sido lagarmente utilizado na investigação das excitações elementares de plasma em semicondutores, particularmente após a invenção do laser (década de 1960).

A resposta de um plasma simples a um estímulo externo (um campo eletromagnético, por exemplo) é descrita pela função dielétrica longitudinal, $\epsilon(\vec{Q}, \omega)$ dependente do vetor de onda $\vec{Q}$ e da freqüência $\omega$, onde $\vec{Q}$ e $\omega$ (em unidades de $\hbar$ ) representam, respectivamente, a transferência de $m o$ mentum e de energia no evento. E como veremos no artigo seguinte (referido como II), a função dielétrica está intimamente relacionada com a seção de choque de espalhamento. Neste artigo calcularemos a função dielétrica do sistema e obteremos expressões relativamente gerais cujos casos particulares recuperam expressões conhecidas na literatura.

\section{FUNÇÃO DIELÉTRICA: FÓRMULA DE LINDHART E CASO GERAL}

No estudo de propriedades ópticas de materiais semicondutores, a função dielétrica é uma grandeza física fundamental a ser conhecida já que quantidades físicas deter- 
minadas experimentalmente, tais como coeficiente de absorção, a refletividade, o espectro Raman, etc, podem ser calculadas a partir do conhecimento da função dielétrica, bem como fornece informações a respeito das excitações elementares do sistema (de quasi-partículas individuais e oscilações coletivas) [3].

Queremos calcular a função dielétrica longitudinal, $\epsilon(\vec{Q}, \omega)$. Em geral, $\epsilon(\vec{Q}, \omega)$ é uma função complexa dada pela expressão de Lindhart $[3,4]$

$$
\begin{aligned}
& \epsilon(\vec{Q}, \omega)=\epsilon_{\infty} \\
& -V(Q) \lim _{s \rightarrow 0} \sum_{\vec{k}} \frac{f(\vec{k}+\vec{Q})-f(\vec{k})}{E(\vec{k}+\vec{Q})-E(\vec{k})-\hbar(\omega+\imath s)},
\end{aligned}
$$

onde $\epsilon_{\infty}$ é a constante dielétrica óptica, $f(\vec{k})$ é a função distribuição de Fermi-Dirac, $V(Q)=4 \pi e^{2} /\left(\mathcal{V} Q^{2}\right)$ é a transformada de Fourier do potencial coulombiano, $e$ a carga eletrônica e $\mathcal{V}$ o volume da amostra; $E(\vec{k})$ é a relação de dispersão da banda, $\hbar$ é a constante de Planck e $\vec{k}$ é o vetor de onda do elétron. Finalmente, $s$ é uma grandeza infinitesimal positiva que garante a continuação analítica da função. Nos nossos cálculos consideraremos uma banda parabólica, i.e., $E(\vec{k})=\hbar^{2} k^{2} /\left(2 m^{*}\right)$, onde $m^{*}$ é a massa efetiva do elétron. Denominaremos as partes real e imaginária da função dielétrica por $\epsilon_{1}(\vec{Q}, \omega)$ e $\epsilon_{2}(\vec{Q}, \omega)$, respectivamente, de maneira que $\epsilon(\vec{Q}, \omega)=\epsilon_{1}(\vec{Q}, \omega)+\imath \epsilon_{2}(\vec{Q}, \omega)$. Transformando a soma em (1) em uma integral pela conhecida regra

$$
\sum_{\vec{k}} \rightarrow \frac{2 \mathcal{V}}{(2 \pi)^{3}} \int d^{3} k
$$

onde o fator 2 no numerador é devido à degenerescência em spin do elétron e $\mathcal{V}$ é o volume do sistema, e utilizando a relação

$$
\lim _{s \rightarrow 0} \frac{1}{X \pm i s}=v \cdot p \cdot \frac{1}{X} \mp i \pi \delta(X)
$$

onde $v . p$. indica o valor principal de uma integral na qual esta relação é usada e $\delta(X)$ é a função delta de Dirac; obtemos as partes real, $\epsilon_{1}(\vec{Q}, \omega)$, e imaginária, $\epsilon_{2}(\vec{Q}, \omega)$, da função dielétrica

$$
\epsilon_{1}(\vec{Q}, \omega)=\epsilon_{\infty}-\frac{\mathcal{V} V(Q)}{4 \pi^{3}} F(\vec{Q}, \omega)
$$

onde

$$
F(\vec{Q}, \omega)=v \cdot p \cdot \int d^{3} k\left[\frac{f(\vec{k}+\vec{Q})-f(\vec{k})}{E(\vec{k}+\vec{Q})-E(\vec{k})-\hbar \omega}\right]
$$

e

$$
\begin{aligned}
\epsilon_{2}(\vec{Q}, \omega)= & -\frac{\mathcal{V} V(Q)}{4 \pi^{2}} \int[f(\vec{k}+\vec{Q})-f(\vec{k})] \times \\
& \times \delta(E(\vec{k}+\vec{Q})-E(\vec{k})-\hbar \omega) d^{3} k .
\end{aligned}
$$

A parte imaginária da função dielétrica está relacionada com o coeficiente de absorção do plasma em semicondutor [5]. A presença da função delta em (6) expressa a conservação de energia.
Podemos reescrever a Eq. (5) como $^{2}$

$$
\begin{aligned}
F(\vec{Q}, \omega) & =\int d^{3} k\left[\frac{f(\vec{k})}{\hbar \omega-[E(\vec{k}+\vec{Q})-E(\vec{k})]}\right] \\
& -\int d^{3} k\left[\frac{f(\vec{k}+\vec{Q})}{\hbar \omega-[E(\vec{k}+\vec{Q})-E(\vec{k})]}\right] .
\end{aligned}
$$

Fazendo a substituição de variável $\vec{k}+\vec{Q}=\vec{k}^{\prime}$ no segundo termo do lado direito da equação acima, e utilizando que $\vec{k}^{\prime}$ é índice mudo obtemos alternativamente que

$$
\begin{aligned}
& F(\vec{Q}, \omega)=\int d^{3} k f(\vec{k}) \times \\
& \times\left[\frac{[E(\vec{k}-\vec{Q})-E(\vec{k})]+[E(\vec{k}+\vec{Q})-E(\vec{k})]}{[\hbar \omega-(E(\vec{k}+\vec{Q})-E(\vec{k}))][\hbar \omega-(E(\vec{k})-E(\vec{k}-\vec{Q}))]}\right] .
\end{aligned}
$$

Utilizando coordenadas esféricas $(k, \theta, \phi)$ e a aproximação de banda parabólica, a equação acima fica

$$
\begin{aligned}
& F(Q, \omega)=\frac{2 \pi \hbar^{2}}{m^{*}} \int_{0}^{\infty} f(k) k^{2} d k \times \\
& \times \int_{-1}^{1} \frac{d u}{\left[\left(\frac{\hbar^{2} k}{m^{*}}\right)^{2} u^{2}-\frac{2 \hbar^{3} \omega k}{m^{*} Q} u+\left(\frac{\hbar^{2} \omega^{2}}{Q^{2}}-\left(\frac{\hbar^{2} Q}{2 m^{*}}\right)^{2}\right)\right]},
\end{aligned}
$$

onde $u=\cos \theta$ e $\theta$ é o ângulo entre $\vec{k}$ e $\vec{Q}$. A parte angular da (9) pode ser calculada utilizando o seguinte resultado ${ }^{3}$

$$
\int \frac{d x}{a x^{2}+b x+c}=\frac{1}{\sqrt{b^{2}-4 a c}} \ln \left|\frac{2 a x+b-\sqrt{b^{2}-4 a c}}{2 a x+b+\sqrt{b^{2}-4 a c}}\right|,
$$

e a Eq. (9) torna-se

$$
\begin{aligned}
F(Q, \omega)= & -\frac{2 \pi m^{*}}{\hbar^{2} Q} \int_{0}^{\infty} f(k) k\left[\ln \left|\frac{k+q_{1}(Q, \omega)}{k-q_{1}(Q, \omega)}\right|\right. \\
& \left.+\ln \left|\frac{k+q_{2}(Q, \omega)}{k-q_{2}(Q, \omega)}\right|\right] d k,
\end{aligned}
$$

onde $q_{1}$ e $q_{2}$ são funções de $Q$ e $\omega$ e valem

$$
q_{1}(Q, \omega)=\frac{Q}{2}-\frac{m^{*} \omega}{\hbar Q}, \quad q_{2}(Q, \omega)=\frac{Q}{2}+\frac{m^{*} \omega}{\hbar Q} .
$$

Vejamos agora o cálculo da parte imaginária: Podemos reescrever a (6) como

$$
\begin{aligned}
& \epsilon_{2}(\vec{Q}, \omega)=\frac{\mathcal{V} V(Q)}{4 \pi^{2}}\left[\int d^{3} k f(\vec{k}) \times\right. \\
& \times \delta(E(\vec{k}+\vec{Q})-E(\vec{k})-\hbar \omega) \\
& \left.-\int d^{3} k f(\vec{k}+\vec{Q}) \delta(E(\vec{k}+\vec{Q})-E(\vec{k})-\hbar \omega)\right] .
\end{aligned}
$$

\footnotetext{
2 Nas equações que se seguem para $F(\vec{Q}, \omega)$ omitiremos o símbolo v.p., ficando este implícito.

3 Em nosso caso $\sqrt{b^{2}-4 a c}=\frac{\hbar^{4} Q k}{m^{*}}>0$
} 
Novamente, fazendo a mudança de variável $\vec{k}+\vec{Q}=\vec{k}^{\prime}$ no segundo termo do lado direito da equação acima ficamos com a expressão

$$
\begin{aligned}
\epsilon_{2}(\vec{Q}, \omega)= & \frac{\mathcal{V} V(Q)}{4 \pi^{2}} \times \\
& \times\left[\int d^{3} k f(\vec{k})[\delta(E(\vec{k}+\vec{Q})-E(\vec{k})-\hbar \omega)]\right. \\
& \left.-\int d^{3} k f(\vec{k})[\delta(E(\vec{k}-\vec{Q})-E(\vec{k})+\hbar \omega)]\right]
\end{aligned}
$$

e, mais uma vez, utilizando coordenadas esféricas e a aproximação de banda parabólica encontramos para $\epsilon_{2}(Q, \omega)$

$$
\begin{aligned}
\epsilon_{2}(Q, \omega)= & \frac{\mathcal{V} V(Q)}{2 \pi} \int_{0}^{\infty} f(k) k^{2} d k \times \\
& \times \int_{-1}^{1} d u\left[\delta\left(\frac{\hbar^{2} k Q}{2 m^{*}} u+\epsilon_{Q}-\hbar \omega\right)\right. \\
& \left.-\delta\left(-\frac{\hbar^{2} k Q}{2 m^{*}} u+\epsilon_{Q}+\hbar \omega\right)\right],
\end{aligned}
$$

onde, como já definido, $u=\cos \theta$ e $\epsilon_{Q}=\hbar^{2} Q^{2} /\left(2 m^{*}\right)$.

Integrando a parte angular de (15) encontramos

$$
\epsilon_{2}(Q, \omega)=\frac{\mathcal{V} V(Q)}{2 \pi} \int_{0}^{\infty} d k f(k) k^{2}\left[I_{1}(k, Q)-I_{2}(k, Q)\right]
$$

onde

$$
I_{1}(k, Q)=\left\{\begin{array}{cc}
\frac{m^{*}}{\hbar^{2} k Q}, & k>\left|q_{1}(Q, \omega)\right| \\
0, & k \leq\left|q_{1}(Q, \omega)\right|
\end{array}\right.
$$

e

$$
I_{2}(k, Q)=\left\{\begin{array}{cc}
\frac{m^{*}}{\hbar^{2} k Q}, & k>\left|q_{2}(Q, \omega)\right| \\
0, & k \leq\left|q_{2}(Q, \omega)\right|
\end{array}\right.
$$

onde $q_{1}$ e $q_{2}$ são definidos pela equação (12).

Para calcularmos a função dielétrica devemos substituir a função distribuição $f(\vec{k})$ em $(11)$ e (16) e calcular as integrais resultantes. Normalmente é encontrado nos livros textos o cálculo para sistemas degenerados, i.e., para temperaturas tais que $T \ll T_{F}$, onde $T_{F}$ é a temperatura de Fermi (ver, por exemplo, [6]). Nestas situações a distribuição de Fermi-Dirac é aproximada por uma função degrau no nível de Fermi; ou então é admitido de inicio pequenos valores de vetor de onda $\vec{Q}$ de maneira que as seguintes aproximações possam ser usadas

$$
\begin{aligned}
f(\vec{k}+\vec{Q})-f(\vec{k}) & \approx \vec{Q} \cdot \nabla_{k} f(\vec{k}) \\
E(\vec{k}+\vec{Q})-E(\vec{k}) & \approx \vec{Q} \cdot \nabla_{k} E(\vec{k})
\end{aligned}
$$

Contudo, no limite de altas temperaturas $\left(T \gg T_{F}\right)$, quando a função de Fermi-Dirac pode ser aproximada por uma distribuição do tipo Maxwell-Boltzmann, o cálculo das integrais é muito mais complicado, particularmente o da Eq. (11) que resulta em funções transcendentais. Não obstante, na próxima seção obteremos expressões analíticas para as partes real e imaginária da função dielétrica que (dentro da aproximação de banda parabólica) é válida para qualquer valor de $Q$. Todavia, devemos lembrar que a aproximação de banda parabólica é inadequada para valores de $Q$ distante do centro da zona de Brillouin mas, para experimentos ópticos, por exemplo, onde os valores envolvidos de $Q$ são pequenos em comparação com o raio da zona de Brillouin, os nossos resultados são totalmente adequados.

\section{CÁLCULO DA FUNÇÃO DIELÉTRICA NO LIMITE DE ALTAS TEMPERATURAS}

Como já dito, no limite de altas temperaturas $\left(T \gg T_{F}\right)$, que é um caso adequado no estudo de semicondutores à temperatura ambiente, a função distribuição de FermiDirac pode ser aproximada por uma distribuição do tipo Maxwell-Boltzmann

$$
f(E(k))=\frac{n \hbar^{3}}{2}\left(\frac{2 \pi \beta}{m^{*}}\right)^{3 / 2} \exp \left(-\frac{\beta \hbar^{2} k^{2}}{2 m^{*}}\right)
$$

onde $n$ é a concentração de portadores e, como usual, $\beta=1 /\left(k_{B} T\right)$ onde $k_{B}$ é a constante de Boltzmann e $T$ é a temperatura do sistema.

Substituindo (21) em (11) temos:

$$
\begin{aligned}
F(Q, \omega)= & -\left(\frac{2 \pi \beta}{\left(m^{*}\right)^{1 / 3}}\right)^{3 / 2} \frac{\pi \hbar n}{Q} \times \\
& \times \int_{0}^{\infty} d k k \exp \left(-\xi^{2} k^{2}\right) \times \\
& \times\left[\ln \left|\frac{k+q_{1}(Q, \omega)}{k-q_{1}(Q, \omega)}\right|+\ln \left|\frac{k+q_{2}(Q, \omega)}{k-q_{2}(Q, \omega)}\right|\right],
\end{aligned}
$$

onde fizemos $\xi^{2}=\beta \hbar^{2} /\left(2 m^{*}\right)$. Na equação acima temos duas integrais do tipo

$$
A=\int_{0}^{\infty} x \exp \left(-\xi^{2} x^{2}\right) \ln \left|\frac{x+q}{x-q}\right| d x
$$

onde $q$ pode ser $q_{1}$ ou $q_{2}$.

Integrando (23) por partes obtemos

$$
A=\frac{1}{\xi^{2}} \int_{0}^{\infty} \frac{\exp (-y x)}{1-x^{2}} d x=\frac{1}{\xi^{2}} I(y)
$$

onde $y=(\xi q)^{2}$. Mas, $I(y)$ satisfaz à seguinte equação diferencial:

$$
\frac{d I(y)}{d y}+I(y)=\frac{1}{2} \sqrt{\left(\frac{\pi}{y}\right)}
$$

cuja solução é:

$$
I(y)=\frac{\sqrt{\pi}}{2} \int_{0}^{y} \frac{\exp (-y) \exp (x)}{\sqrt{x}} d x
$$

que pode ser reescrita como

$$
\begin{aligned}
I(y) & =\sqrt{\pi} \exp (-y) \int_{0}^{\sqrt{y}} \exp \left(x^{2}\right) d x \\
& =\sqrt{\pi} \exp \left[-(\xi q)^{2}\right] \int_{0}^{\xi q} \exp \left(x^{2}\right) d x .
\end{aligned}
$$

Assim podemos escrever (23) como,

$$
A=\frac{\sqrt{\pi}}{\xi^{2}} D(y)
$$

onde $D(y)$ é a chamada integral de Dawson [7]

$$
D(y)=\exp \left(-y^{2}\right) \int_{0}^{y} \exp \left(x^{2}\right) d x
$$


A Figura 1 mostra o gráfico de $D(y)$ e seus casos limites para $y \ll 1$ e $y \gg 1$.

Portanto, (22) pode ser escrita como

$$
F(Q, \omega)=-\frac{2^{5 / 2} \pi^{3}\left(m^{*} \beta\right)^{1 / 2} n}{\hbar Q}\left[D\left(y_{1}\right)+D\left(y_{2}\right)\right],
$$

onde

$$
\begin{aligned}
& y_{1}(Q, \omega)=\left(\frac{\beta \hbar^{2}}{2 m^{*}}\right)^{1 / 2}\left[\frac{Q}{2}+\frac{m^{*} \omega}{\hbar Q}\right], \\
& y_{2}(Q, \omega)=\left(\frac{\beta \hbar^{2}}{2 m^{*}}\right)^{1 / 2}\left[\frac{Q}{2}-\frac{m^{*} \omega}{\hbar Q}\right] .
\end{aligned}
$$

Substituindo (30) em (4) obtemos para a parte real

$$
\epsilon_{1}(Q, \omega)=\epsilon_{\infty}\left[1+\left(\frac{2 m^{*}}{\beta}\right)^{1 / 2} \frac{k_{D H}^{2}}{\hbar Q^{3}}\left[D\left(y_{1}\right)+D\left(y_{2}\right)\right]\right],
$$

onde

$$
k_{D H}^{2}=\frac{4 \pi e^{2} n}{\epsilon_{\infty} k_{B} T}
$$

é o vetor de onda de Debye-Hückel.

Da mesma forma, substituindo (21) em (16) encontramos para a parte imaginária

$$
\epsilon_{2}(Q, \omega)=\epsilon_{\infty}\left(\frac{\pi m^{*}}{2 \beta}\right)^{1 / 2} \frac{k_{D H}^{2}}{\hbar Q^{3}}\left[\exp \left(-y_{2}^{2}\right)-\exp \left(-y_{1}^{2}\right)\right] .
$$

As equações (32) e (34) são expressões analíticas gerais, válidas para qualquer valor de magnitude de vetor de onda $Q$. Além do mais as expressões acima têm paridade definida: a parte real é par em $\omega$, ou seja, para $Q$ fixo $\epsilon_{1}(Q,-\omega)=\epsilon_{1}(Q, \omega)$, enquanto que a parte imaginária é ímpar em $\omega$, i.e., $\epsilon_{2}(Q,-\omega)=-\epsilon_{2}(Q, \omega)$, o que confirma o conhecido resultado da eletrodinâmica dos meios materiais [5].

Podemos ter uma visão física mais profunda da função dielétrica analisando os casos particulares das expressões acima. O que faremos a seguir.

\section{FUNÇÃO DIELÉTRICA: CASOS LIMITES}

Mostraremos agora que as expressões normalmente encontradas na literatura são casos particulares do nosso resultado.

\section{A. Caso estático; fenômeno de blindagem}

Consideremos o caso limite $\omega \rightarrow 0$, que determina a resposta do sistema a uma perturbação estática. Neste caso, de (31) temos que

$$
y_{1}=y_{2}=\left(\frac{\beta}{2 m^{*}}\right)^{1 / 2} \frac{\hbar Q}{2},
$$

e vemos de (34) que $\epsilon_{2}(Q, 0)=0$, enquanto a parte real fica

$$
\epsilon_{1}(Q, 0)=\epsilon_{\infty}\left[1+2\left(\frac{2 m^{*}}{\beta}\right)^{1 / 2} \frac{k_{D H}^{2}}{\hbar Q^{3}} D\left(y_{1}\right)\right] .
$$

Adicionalmente, se considerarmos o limite de grandes comprimentos de onda, ou seja, $Q \rightarrow 0$, temos que (ver Apêndice) $D\left(y_{1}\right) \approx y_{1}$, onde $y_{1}$ é dado por (35). Utilizando este resultado na equação acima, obtemos

$$
\epsilon_{1}(Q, 0)=\epsilon_{\infty}\left[1+\frac{k_{D H}^{2}}{Q^{2}}\right] .
$$

Mostraremos agora que o efeito de blindagem que surge em um sistema de muitos corpos pode ser descrito pela função dielétrica estática $\epsilon(Q, 0)$.

O potencial efetivo (blindado) que atua no plasma é

$$
V_{e f}(Q, 0)=\frac{V_{o}(Q, 0)}{\epsilon(Q, 0)}=\frac{Q^{2}}{Q^{2}+k_{D H}^{2}} \frac{V_{o}(Q, 0)}{\epsilon_{\infty}},
$$

onde $V_{o}(Q, 0)$ é o potencial coulombiano sem blindagem. Consideremos uma impureza de carga $q$ colocada no material. Ela dá origem a um potencial da forma

$$
V_{o}(Q, 0)=\frac{4 \pi q}{\mathcal{V} Q^{2}} .
$$

Obtemos então para o potencial efetivo (blindado) que atua no plasma

$$
V_{\text {efe }}(Q, 0)=\frac{V(Q, 0)}{\epsilon(Q, 0)}=\frac{4 \pi q}{\mathcal{V} \epsilon_{\infty}} \frac{1}{Q^{2}+k_{D H}^{2}}
$$

ou, em termos de distância, tomando a transformada de Fourier da equação acima

$$
V(r)=\frac{q}{r} \exp \left(-k_{D H} r\right),
$$

que resulta em um potencial de curto alcance (tipo Yukawa), conseqüência dos efeitos de blindagem ou polarização da carga eletrônica. Isso explica porque a força de Coulomb, mesmo sendo de longo alcance, é pouco efetiva em um gás de elétrons denso, o que justifica o modelo do elétron independente.

\section{B. Limite da altas freqüências $\omega \gg \hbar Q^{2} /\left(2 m^{*}\right)$; oscilação de plasma}

\section{Parte real}

A expansão assintótica $(y \gg 1)$ para a integral de Dawson é dada por [conforme Eq. (A.9)]

$$
D(y) \approx \frac{1}{2 y}\left(1+\frac{1}{2 y^{2}}+\ldots\right) .
$$

Utilizando a expressão acima em (32) encontramos depois de alguns cálculos

$$
\epsilon_{1}(Q, \omega)=\epsilon_{\infty}\left[1-\frac{\omega_{p}^{2}}{\omega^{2}}\left(1+\frac{3 Q^{2}}{\beta m^{*} \omega^{2}}\right)\right],
$$

onde

$$
\omega_{p}^{2}=\frac{4 \pi e^{2} n}{\epsilon_{\infty} m^{*}}
$$

é a freqüência de plasma do sistema. 
A partir da Eq. (43) podemos obter a relação de dispersão. As excitações elementares do sistema são as raízes da parte real da função dielétrica, então da Eq. (43) obtemos

$$
\omega^{2}=\omega_{p}^{2}\left(1+\frac{3 Q^{2}}{\beta m^{*} \omega^{2}}\right) .
$$

A freqüência que aparece entre colchetes pode ser identificada com a freqüencia de plasma sem introduzir erro significativo. Logo

$$
\omega^{2}=\omega_{p}^{2}\left(1+\frac{3 Q^{2}}{\beta m^{*} \omega_{p}^{2}}\right),
$$

ou ainda, em termos da velocidade quadrática média

$$
v_{q m}^{2}=<v^{2}>=\frac{3 k_{B} T}{m^{*}}
$$

a eq.(46) resulta em

$$
\omega^{2}=\omega_{p}^{2}\left[1+\frac{<v^{2}>Q^{2}}{\omega_{p}^{2}}\right],
$$

que é a relação de dispersão procurada.

Se fizermos $Q \rightarrow 0$ em (43) teremos que

$$
\epsilon_{1}(0, \omega)=\epsilon_{\infty}\left(1-\frac{\omega_{p}^{2}}{\omega^{2}}\right)
$$

Assim, ao contrário do caso anterior (caso estático), onde o potencial coulombiano era blindado, agora vemos que a função dielétrica acentua fortemente esta interação próximo a $\omega=\omega_{p}$. E mais, vemos que uma flutuação na densidade do sistema pode ser criada mesmo na ausência de uma perturbação externa. Isto corresponde a um novo auto-estado do sistema, que descreve uma oscilação coletiva chamada de plasmon. Qual a origem física do plasmon? Se causamos um distúrbio na densidade de carga do sistema em um certo instante, os elétrons se moverão a fim de blindar esta carga; mas, provavelmente, eles passarão da posição de equilíbrio e assim aparecerão forças restauradoras que podem fazer com que as oscilações persistam indefinidamente.

\section{Parte imaginária}

No limite que estamos considerando, $\omega \gg \frac{\hbar Q^{2}}{\left(2 m^{*}\right)}$, a Eq. (31) fica

$$
y_{1}^{2}=\frac{\beta m^{*} \omega^{2}}{2 Q^{2}}+\frac{\beta \hbar \omega}{2}, \quad y_{2}^{2}=\frac{\beta m^{*} \omega^{2}}{2 Q^{2}}-\frac{\beta \hbar \omega}{2} .
$$

Utilizando (50) em (34), obtemos

$$
\begin{aligned}
\epsilon_{2}(Q, \omega)= & \epsilon_{\infty}\left(\frac{2 \pi m^{*}}{\beta}\right)^{1 / 2} \frac{k_{D H}^{2}}{\hbar Q^{3}} \times \\
& \times \exp \left(\frac{-\beta m^{*} \omega^{2}}{2 Q^{2}}\right) \sinh \left(\frac{\beta \hbar \omega}{2}\right) .
\end{aligned}
$$

Adicionalmente, se considerarmos que $k_{B} T \gg \hbar \omega / 2$, obtemos a expressão clássica

$$
\epsilon_{2}(Q, \omega)=\epsilon_{\infty}\left(\frac{\pi m^{*} \beta}{2}\right)^{1 / 2} \frac{k_{D H}^{2}}{Q^{3}} \omega \exp \left(\frac{-\beta m^{*} \omega^{2}}{2 Q^{2}}\right) .
$$

A parte imaginária da função dielétrica está relacionada com a dissipação do sistema.

\section{CONSIDERAÇÕES FINAIS}

Nosso objetivo neste trabalho foi obter expressões analíticas para as partes real e imaginária da função dielétrica longitudinal para um plasma em estado sólido no limite de altas temperaturas $\left(T \gg T_{F}\right)$, situação em que podemos substituir a função distribuição de Fermi-Dirac por uma do tipo Maxwell-Boltzmann.

A partir das expressões gerais podemos obter casos particulares que ilustram de maneira significativa aspectos físicos do sistema. Desse modo, vemos que as excitações elementares do sistema podem ser divididas em duas partes:

1. Uma parte representando oscilações coletivas (oscilações de plasma) - e portanto relacionada com o comportamento coletivo do sistema - produzida pelo efeito de longo alcance da força coulombiana que leva às interações simultâneas entre as partículas.

2. A outra parte está associada com o comportamento individual das partículas, originado do movimento aleatório das mesmas.

Apesar de não termos feito explicitamente nenhuma hipótese restritiva sobre os valores assumidos por $Q$, utilizamos nos nossos cálculos a aproximação de banda parabólica. Isso significa que, na prática, devemos nos restringir a valores de $Q$ próximos ao centro da zona de Brillouin. Mas, como já ressaltado, em experimentos ópticos (no visível) essa condição é plenamente satisfeita, o que torna os nossos resultados interessantes, conforme veremos no artigo II.

\section{APÊNDICE: FORMAS ASSINTÓTICAS DA INTEGRAL DE DAWSON}

Para obtermos os casos particulares da parte real da função dielétrica precisamos conhecer o comportamento da integral de Dawson, $D(y)$, nos casos em que a variável $y$ assume valores muito grandes ou muito pequenos.

Para $y \ll 1$, a Eq. (29) pode ser escrita como

$$
D(y) \approx\left(1-y^{2}\right) \int_{0}^{y}\left(1+x^{2}+\ldots\right) d x=\left(1-y^{2}\right)\left(y+\frac{y^{3}}{3}\right) .
$$

Desprezando termos de ordem superior a dois obtemos

$$
D(y) \approx y
$$

Assim, próximo a origem $D(y)$ tem um comportamento linear (Figura 1).

Vejamos agora o comportamento assintótico de $D(y)$.

Inicialmente, consideremos a integral de probabilidade definida para uma variável complexa $z$ de acordo com [8]

$$
\Phi(z)=\frac{2}{\sqrt{\pi}} \int_{0}^{z} \exp \left(-t^{2}\right) d t
$$

avaliada ao longo de uma trajetória arbitrária que liga a origem ao ponto $t=z$. 
É fácil ver que a integral de Dawson e a integral de probabilidade estão relacionadas. Escolhendo um segmento do eixo imaginário como um caminho de integração $z=\imath y \mathrm{e}$ fazendo a substituição $t=\imath u,($ A.3) torna-se

$$
\Phi(\imath y)=\frac{\sqrt{\pi}}{2 \imath} \exp \left(-y^{2}\right) \Phi(y)
$$

Comparando (29) com (A.4) podemos escrever $D(y)$ como

$$
D(y)=\frac{2 \imath}{\sqrt{\pi}} \int_{0}^{z} \exp \left(u^{2}\right) d u .
$$

mas, $\Phi(z)$ tem a seguinte expansão assintótica [8]

$\Phi(z)=1-\frac{\exp \left(-z^{2}\right)}{\sqrt{\pi} z}\left[1+\sum_{n=1}^{\infty}(-1)^{n} \frac{1 \cdot 3 \cdot \ldots \cdot(2 n-1)}{\left(2 z^{2}\right)^{n}}\right]$.

Para $z=\imath y$ (A.6) torna-se

$$
\Phi(\imath y)=1-\frac{\exp \left(y^{2}\right)}{\sqrt{\pi} \imath y}\left[1+\sum_{n=1}^{\infty} \frac{1 \cdot 3 \cdot \ldots \cdot(2 n-1)}{\left(2 z^{2}\right)^{n}}\right] .
$$

Substituindo (A.7) em (A.5) obtemos a expansão assintótica para a integral de Dawson

$D(y)=\frac{\sqrt{\pi}}{2 \imath} \exp \left(-y^{2}\right)+\frac{1}{2 y}\left[1+\sum_{n=1}^{\infty} \frac{1 \cdot 3 \cdot \ldots \cdot(2 n-1)}{\left(2 y^{2}\right)^{n}}\right]$.

Para $y \rightarrow \infty$ (A.8) reescreve-se

$$
D(y)=\frac{1}{2 y}\left[1+\sum_{n=1}^{\infty} \frac{1 \cdot 3 \cdot \ldots \cdot(2 n-1)}{\left(2 y^{2}\right)^{n}}\right],
$$

considerando apenas o primeiro termo na expressão acima, obtém-se

$$
D(y)=\frac{1}{2 y}\left(1+\frac{1}{2 y^{2}}\right) .
$$

Vemos da Figura 1 que (A.8) é uma excelente aproximação para a integral de Dawson para $y \geq 1,5$.

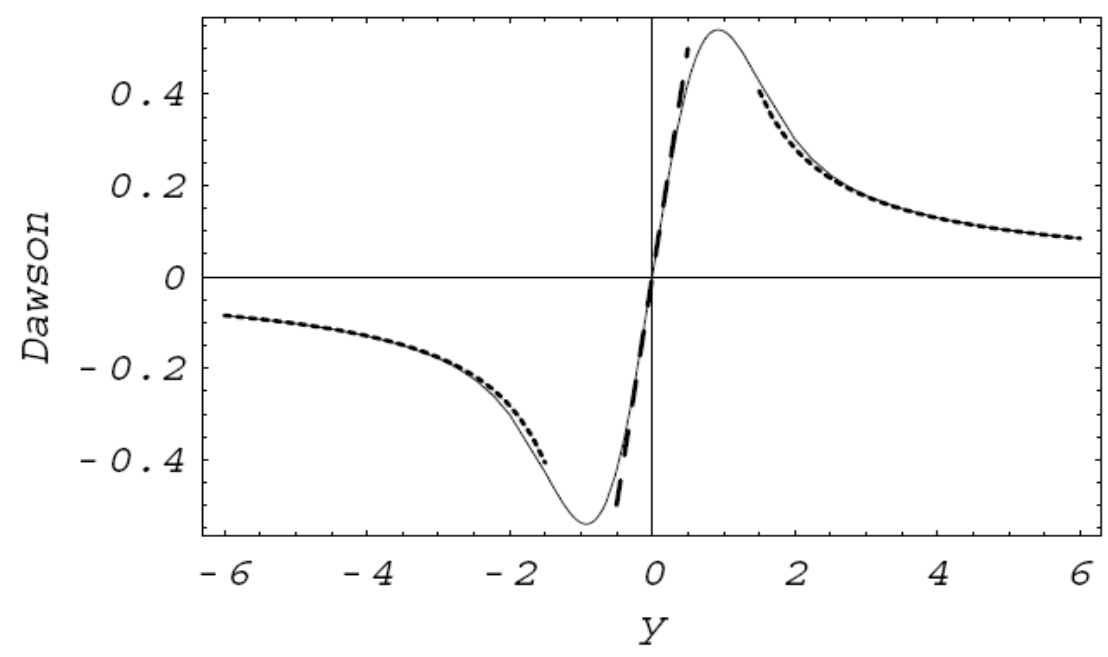

Gráfico de $D(y)$ [Eq. (29)] (linha contínua) e seus casos limites: para $y \ll 1$ [Eq. (A.1)] (linha pontilhada), para $-1<y<1$ [Eq. (A.2)] (linha tracejada) e para $y \gg 1$ [Eq. (A.9)] (linha pontilhada).

[1] P.M. Platzman and P.A. Wolff; Waves and Interactions in Solid State Plasmas, In F. Seitz, D. Turnbull and H. Ehrenrich editors, Solid State Physics, Suppl. 13, Academic Press, NY (1973).

[2] R. Luzzi, A.R. Vasconcellos and J.G.P. Ramos; Predictive Statistical Mechanics: A Nonequilibrium Ensemble Formalism, Kluwer Academic, Dordrecht (2002).

[3] S. Nakajimal et al; The Physics of Elementary Excitations, Springer-Verlag, Berlin (1980).

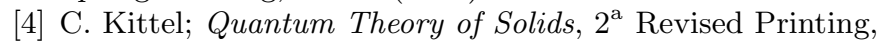

John Wiley and Sons, NY (1987).

[5] L. Landau and L.E. Lifshitz; Electrodynamics of Continuous Media, Addison-Wesley (1984).

[6] G.D. Mahan; Many-Particle Physics, $3^{\text {a }}$ Ed., Kluwer Academic, NY (2000).

[7] M. Abramovitz and I.A. Stegun; Handbook of Mathematical Functions, Dover, NY (1970).

[8] N.N. Lebedev; Special Functions and Their Applications, Dover, NY (1972). 